\title{
Research on WLAN Networking Mode Based on Ad Hoc
}

\author{
Zengyou Sun \\ Department of Information Engineering \\ Northeast Dianli university \\ Jilin, China \\ Sunzengyou@163.com
}

\author{
Chuanhui Hao \\ Department of Information Engineering \\ Northeast Dianli university \\ Jilin, China \\ hchh518666@163.com
}

\begin{abstract}
To enhance the WLAN data transmission capacity and improve network coverage, with ad hoc relay nodes introduced in WLANS, proposed a network structure, which can construct multi-hop network and realize ad-hoc networking. This paper first introduced network model structure of WLAN multiple hops ad hoc, then put forward a fast networking scheme of ad hoc network based on IEEE802.15.4 protocol standard, and then network design. The scheme, with low power consumption and fast networking, can effectively make up for the WLAN network coverage.
\end{abstract}

Keywords-WLAN; networking structure; ad hoc network; the IEEE802.15.4 protocol standard;

\section{INTRODUCTION}

Future WLAN network is a heterogeneous mixture network with a set of wireless access and mixed services. With the support of converged network, users can switch between different systems seamlessly, thus enjoying high speed multimedia services. So providing seamless service to meet customers' demand is the mark of successful wireless communication systems in heterogeneous environments. In addition, there are many communication blind zones for WLAN in cities, due to radio waves attenuation and shadow shade. Introducing ad hoc relay nodes in WLAN can enhance data transmission capacity and improve network coverage.

Wireless Ad Hoc networks has gained researchers' great attention, for flexible networking and not needing infrastructure support, in recent years. To say that there is still much work to do to realize wireless Ad Hoc network large-scale application, if the large-scale application of wireless Ad Hoc network has a lot of work to do, the wireless Ad Hoc network in small areas has entered testing phase in few years. These wireless Ad Hoc networks at testing stage, are closely related to WLAN/WPAN, which contains support for Ad Hoc networking modes. Therefore, Ad Hoc network is also called WLAN/WPAN Ad Hoc network.

\section{WLAN AD HOC NETWORKING}

\section{A. WLAN/WPAN Ad Hoc network model}

On the principle of WLAN subject to Ad hoc, multiple hops self-organizing WLAN/WPAN Ad hoc network model is formulated through expanding WLAN of direct connection. Mobile terminal has two wireless communication module, which are use to realized WLAN air interface protocol and Ad hoc network respectively. Owning two air interface, user terminal has the ability of forwarding data to other users. The multi-hop WLAN/WPAN Ad Hoc network model is shown in figure 1.

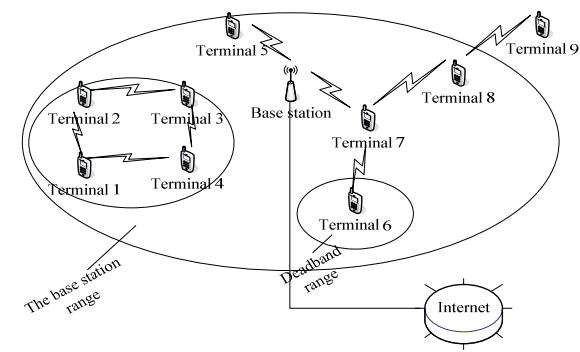

Fig.1 multi-hop WLAN/WPAN Ad Hoc network model

In the system, the user can complete the local communication by the self-organizing way (such as terminal 1 to 4). Liking base station as the center control in WLAN network, it communicate by directly the base station (such as terminal 5), and it can be also by other terminals for data relay to the base station communication (such as terminal 6 and 9). The introduction of Ad hoc network in WLAN, the communication mode of the network topology, the type of the user terminal and the user can use had changed. There are three kinds of communication mode of WLAN [5],such as communication mode from WLAN communication single, evolved into a self-organizing Ad hoc networks and mixed mode. WLAN Ad Hoc network has the following characteristics:

1) Make up the defect coverage. It will have some "dead zone" in the coverage area of WLAN base station control center communication system. There are two types of these dead: a) absolutely without covering, any signal is not reachable. b) dead area of high-speed service unreachable, but the synchronous signal, signaling and low bit rate speech traffic signals can be up to. Since the introduction of terminal communication organization Ad hoc can effectively make up for the above two kinds of coverage defect. It is dead within the terminal 6 in figure 1, and it cannot be directly communicated with the base station, but it can be transmitted through the terminal 7. Many the 
cell edge users was unable to establish a communication link with the base station because of distance and transmission power. The network can make the user terminal in the cell coverage and forwarding access station by other users of relay, which obtain cellular system service and the formation of cell coverage expansion. For example, figure 1 in the coverage of base station and the terminal 9, it cannot be directly communicated with the base station, but through the terminal 7 and terminal 8 of the multi-hop com2.1.2munication.

2) formation of $L A N-W L A N$. In the multi-hop selforganizing network, users can use self-organization, dynamically composed of local area network and the local service transmission. The network is called self-organizing network Ad hoc-WLAN, figure 2 terminal 1 to 4 on the formation of a Ad hoc-WLAN network. So the local service terminal area can be directly through the relay terminal between forwarding to complete, not through the base station to spread across the network, thereby reducing the occupation of the cellular radio channel, to ease the bottleneck station. In addition, when there is no infrastructure support or infrastructure destroyed still can support the communication degree in local region.

\section{B. Ad hoc network structure}

Ad hoc networks generally have two kinds of structure: flat structure and hierarchical structure[6]. The plane structure as shown in Figure 2, all nodes are equal in the planar structure, so it is also called peer-to-peer structure.

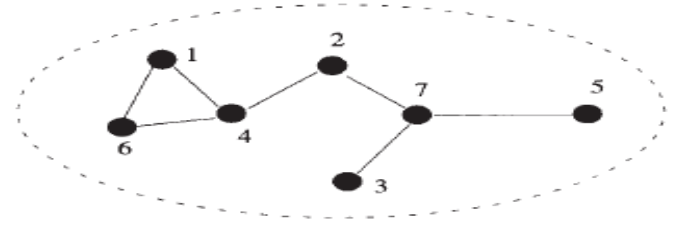

Fig. 2 plane structure

The plane structure of network is simple, no need to maintain any structure. The general existence of multiple paths between the source and the destination station, multiple paths can be used to achieve load sharing, it also can choose the appropriate path for different types of traffic. All points are equal in network, and the bottleneck does not exist in principle, so it is more robust. Coverage of nodes is relatively small and relatively safe in the planar structure. The biggest drawback is the limited scale planar structure network. In the plane structure, each node needs to know to all the other nodes in the routing. Due to the mobility of nodes, the routing information to the large number of control messages to maintain these dynamic changes. Network scale is the larger, so the route maintenance overhead is also the greater. When the network size increases to a certain extent, all of the bandwidth may be routing protocol consumed. So the plane structure network scalability.
Network hierarchical structure is divided into clusters, and each cluster is composed of a cluster head and a plurality of cluster members, and the cluster head to form a network. A high level of the network can be formed at a higher level of network clustering. In the cluster head nodes and cluster member changes, is still the automatic network, composed of cluster head nodes are responsible for data forwarding among clusters. Hierarchical structure according to the different hardware configuration and can be divided into single frequency and multi-frequency hierarchical classification, as shown in figure 3.

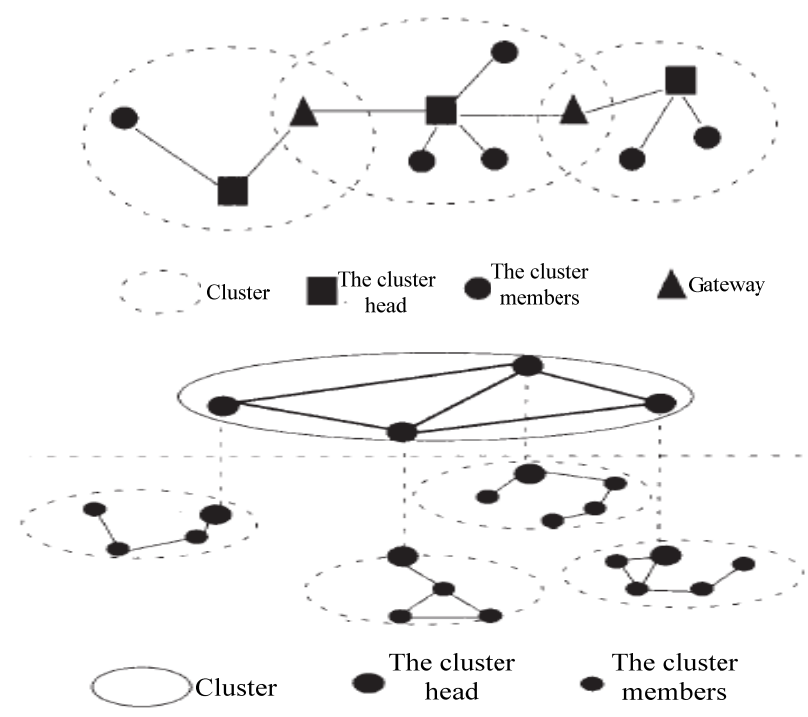

Figure 3 hierarchical structure

1) Single frequency hierarchical structure. Single frequency network is only one communication frequency, and all nodes use the same frequency. The cluster head communication is required between the gateway nodes, which gateway nodes belong to the same two clusters. All nodes use the same frequency communication in single frequency network. In order to realize the communication between cluster heads,it needs a gateway node supporting (also belongs to the two cluster node).

2) Multi-frequency hierarchical structure. Multifrequency hierarchical network is the lower node and small communication range, and advanced node communication range, such as the two level network, a frequency communication with the cluster member nodes and cluster member, a frequency of communication with the cluster head nodes, keep the communication between cluster heads with another frequency.Because a single node distance is limited, and communication within each cluster can be a single jump. It can also be multi-hop communication, and the information will be transmitted to the superior network once the information to cluster head. The network can use higher transmission bandwidth, or it can be superior to the network and it directly connect to a wired network. The 
network is divided into clusters in the hierarchical structure, such as each cluster consists of a cluster head and a plurality of cluster members. In the high level network and clustering, the formation of a greater level of the network is until the most advanced. A hierarchical structure, cluster head node is responsible for forwarding data between clusters. The frequency of a hierarchical network, different level by the communication of different frequency. The communication range of the lower nodes is small, and the top node to cover a large range. Advanced node is at multiple levels, multiple frequency, and it realizes the communication of different levels with different frequency.

\section{WLAN AD HOC NETWORK BASED ON IEEE802.15.4 STANDARD}

\section{A. IEEE802.15.4 Network analysis}

In IEEE802.15.4 network process[4], the other nodes to join the network process after the PAN coordinator starting, and it can be divided into two parts: the channel scanning and requesting to connect :

(1) Channel scan is to choose a less disturbance of logical channel, which can be preset logical channel LAN in needing of fast networking and wireless interference fewer occasions, thus omitting the process of channel scanning, speeding up the network composition.

(2) The coordinator sends a connection to request the LAN after obtaining LAN information, apply to join the LAN. The MAC transmits is an acknowledgment to the nodes in the request state frame layer LAN coordinator, and it primitives to the upper transmission at the same time connection request, which identifies the node connection request. The coordinator of upper MAC layer receives instructions. According to their specific circumstances, it decides whether or not to approve the connection in the long a Response Wait Time symbol period, if approved, then the coordinator to request device is assigned a short address, and sent to the node contains a new address and connection state of the connection response command in non-beacon mode, and a node must wait for the a Response Wait Time symbol periods, to send a data request attempts to get a connection response command.

To realize the distribution of short address, identifies the ID nodes and node corresponding is short in the application of fast network. Setting in a short address prior, node can send directly data short address to set the request, without the need for a process by connection requests. The IEEE802.15.4 channel is divided into 32 channel page (Channel Page), which each channel page uses different modulation mode. The latest standard uses 3channel page and the remaining 29 channel page retention. In each channel page and different logical channels, each logical channel has their operating frequency. For example, the 868 $\mathrm{MHz}$ has 1logical channel, and $915 \mathrm{MHz}$ has 10 logical channel, and $2450 \mathrm{MHz}$ has 16 logical channel.
B. Standard of Ad hoc networking scheme in IEEE802.15.4

Because IEEE802.15.4 supports peer-to-peer network[7], which can build a Mesh network based on point to point, as shown in Figure 4, the function of each node in the network is the same.

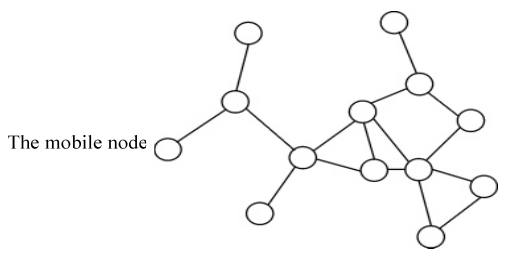

Fig. 4 based on point-to-point network formed by Mesh network

It is shown in Figure 5 based on The formation of IEEE802.15.4 Ad Hoc network. Nodes need to initialize the IEEE802.15.4protocol stack and network layer[2, 3]. The initialization is completed, and each node will set up its own LAN ID, logical channel and short address to a prearranged value in the network. Nodes need to communicate, and need to establish routing information.

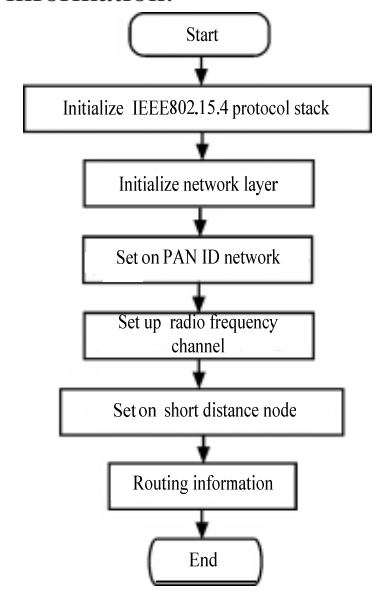

Fig. 5 Ad Hoc network based on IEEE802.15.4

The network layer provides two types of services: network layer data service and network management services. These two kinds of service entity are respectively called NLDE and NLME in the network layer. The two service entity provides interface for upper, also have internal network interface layer between two entities. NLME internal interfaces provided by NLDE, in order to complete some management functions. The reference model of node software as shown in figure 6.

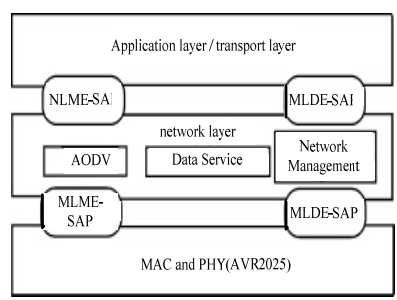

Fig. 6 software reference model 
It may send 3 frames in the network layer: a layer to network layer to a node sends data frame; the network layer due to the path can use can not find, need to broadcast RREQ frame starts the route discovery process; the network layer of the RREQ message is received, meet the conditions required to send the RREP message to the source node. When the network layer to send data frames, the active path will first arrive at the destination node in the local routing table lookup. If you find the path, the data frames transmitted along the path; if no path is found, will be the first to send the data frame buffer, and then calls the routing module, generating a RREQ message, starting the routing discovery process. When the network layer receives a data frame, the destination node first checks whether the data frame for this node. If the node, the network layer of the frame head of the data frame removed, transmits the data to a destination node; if not for the nodes, the network layer in the local routing table look up to a destination node in the next hop. If it find a next hop address, the data frame is forwarded to the next hop to continue; if there is no next hop address, network layer needs to generate the RREQ message, start the routing discovery process. When the network layer receives a RREQ message, RREP message or other command frame, it will be handed over to the routing module for processing. The micro grid adjacent using different frequency bands or time frequency code (here referred to as channel) in order to reduce the micro interference between networks. Each micro grid is composed of a plurality of terminal DEV, where it is a higher priority to the DEV as a micro grid PNC, and it is responsible for the network management. The same within the micro grid DEV for end-to-end communication. Micro media access control network based on the timing of super frame structure, as shown in figure 7

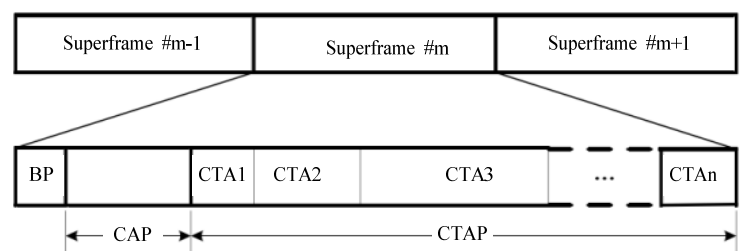

Fig. 7 Micro media access control network based on the timing of super frame structure

The above shows, MAC super frame consists of three parts:

(1) Beacon period (BP) micro grid PNC used to broadcast beacon,beacon frames containing the current super frame timing information, slot allocation information and other information management;
(2) Competitive access period (CAP) during this period, the terminal by means of competition, for example, CSMA/CA contention channel. It can be used to exchange the command frame and asynchronous data;

(3) Channel time allocation period (CTAP) no competition, the micro grid PNC according to the load distribution slot DEV application and the network bandwidth to apply for DEV. It can be used to exchange the command frame, asynchronous data and synchronous data stream;

\section{CONCLUSION}

Providing seamless service to meet customers' demand is the mark of successful wireless communication systems in heterogeneous environments. With ad hoc relay nodes introduced in WLANs can enhance the data transmission capacity and improves network coverage. The Ad Hoc networking scheme based on IEEE802.15.4 standards is proposed to achieve the above goal. The designed network layer based on IEEE802.15.4 can provide data service and management service function interface. While the research of WLAN multi-hop ad Hoc network technology is still in the initial stage, mixed network capacity, user identity authentication ,security, QoS security are needed to be further studied.

\section{REFERENCES}

[1] Perkins C E.Ad Hoc Networking: An introduction[M].Boston: AddisonWesley Longman, 2001.30-36.

[2] Jubin J,Tornow J D. The DARPA packet radio network protocol[J]. Proc.of the IEEE, 1987, 75(1):21-32.

[3] Perkins C E,Belding-Royer E M. Ad hoc On-Demand Distance Vector Routing [C]//Proceedings of Mobile Computing System and Applications, California: [s.n.],1999:90-100.

[4]Fanliang Meng, Yigang Han. Research on fast networking in IEEE802.15.4 [J] Journal of Nankai University: Natural Science Edition, 2010, 43(3): 61-65.

[5] Huang Liang. Study on mobile routing technology in wireless sensor network [D]. Xi'an: Northwestern Polytechnical University,2011. 42-56.

[6] Zhifeng Zhao,Shaoren Zheng. Research on hoc Ad network architecture Telecommunications Science 2001 first period .2010.14-17

[7] LAN/MAN Standards Committee. IEEE Std 802.15.4TM-2006.IEEE Standard for Information technology Telecommunications and information exchange between systems Local and metropolitan area networks Specific requirements Part 15.4:Wireless Medium Access Control ( MAC ) and Physical Layer ( PHY ) Specifications for LowRate Wireless Personal Area Networks ( WPANs ) [S].NewYork: IEEE, 2006.

[8] Perkins C E, Belding-Royer E M. Ad hoc On-Demand Distance Vector Routing [C]//Proceedings of Mobile Computing System and Applications, California: [s.n.], 1999:90-100. 